\title{
New insights about myofibrillar myopathies: the role of metalloproteinases 2 and 9 in the pathogenesis
}

Alzira Alves de Siqueira Carvalho

Faculdade de Medicina do ABC

\section{Vinicius Gomes Silva}

Faculdade de Medicina do ABC

\section{Roseli Corazzini}

Faculdade de Medicina do ABC

Alan Patricio Silva ( $\nabla$ alanpatricio.fmabc@gmail.com )

Faculdade de Medicina do ABC https://orcid.org/0000-0003-2918-0060

Emmanuelle Lacene

Hopital Pitie-Salpetriere Departement de Neuropathologie

\section{Research article}

Keywords: MMP-2, MMP-9, Myofibrillar myopathy, muscle biopsy, metalloproteinase.

Posted Date: April 3rd, 2020

DOI: https://doi.org/10.21203/rs.2.20954/v2

License: (c) (1) This work is licensed under a Creative Commons Attribution 4.0 International License. Read Full License 


\section{Abstract}

Background There are few reports suggesting that gene expression and activation of various matrix metalloproteinases (MMPs) are deregulated. MMP-2 and MMP-9 represent the two MMPs, which degrade type IV collagen, the component of basement membrane. Methods We analyzed the involvement of gelatinases, MMP-2 and MMP-9, in the pathogenesis of myofibrillar myopathy (MFM). Muscle specimens from 23 patients well diagnosed with MFM, were immunostained by MMP-2 and MMP-9. We analyzed qualitatively the immunoexpression in three compartments: subsarcolemmal (SSC), intracytoplasmic (ICC) and perinuclear (PNC). Results 95,7\% and 100\% samples showed MMP-2 and MMP-9 upregulation ICC, respectively. PNC showed MMP-2 (82,6\%) and MMP-9 $(8,7 \%)$ regulation $(p<0.001)$. SSC and ICC did not present statistical significance. There was no correlation between mutated gene and immunohistochemical pattern distribution. Conclusion Our results suggest that MMP-2 and/or MMP-9 could participate in the pathomechanism of MFM, causing damage of sarcomere and deposition of protein aggregates.

\section{Background}

Since the discovery of metalloproteinases and its functions in 1962, they have been identified as extracellular matrix (ECM) proteinases responsible for transforming the matrix and degrading its components [1]. However, the discovery of an intracellular function of MMP-2 challenged this idea corroborating that MMP-2 not only repairs the ECM but also acts intracellularly in ischemia-reperfusion in myocardium by degrading troponin I, contributing therefore, to cardiac dysfunction $[1,2,3,4]$.

Regarding the role of MMPs in some myopathies, there are few reports suggesting that stimulation of various MMPs and also gene expression is deregulated. Whilst MMP-2 and MMP-9 are likely involved in the pathology of dystrophin-deficient skeletal muscle [5,6], MMP-9 is implicated in the inflammatory myopathies during the process of elimination of necrotic fibers and muscle degeneration and MMP-2 may be associated with the process of reconstruction of ECM during muscle regeneration and fiber growth $[7,8]$.

Myofibrillar myopathies (MFM), a heterogeneous group of hereditary or sporadic skeletal and cardiac muscle disorders of sarcomere, are still a challenge in terms of our understanding about the pathogenesis [9]. Many different components are involved in the cascade of events occurring in muscle fibers in these disorders, as mutant proteins, a defective ubiquitin - proteasome system and oxidative stress [10]. Nevertheless, there are no previous studies correlating the activation of MMPs and MFM.

In order to determine other components engaged in this pathogenic process we decided to examine the involvement of the gelatinases MMP-2 and MMP-9 in in muscle fibers in MFM.

\section{Methods}


Muscle biopsies from 23 well-characterized patients with a previous diagnosis of MFM were studied. The diagnosis was obtained by clinical findings, morphological and ultrastructural aspects of muscle biopsies, and genetic studies. Control muscles specimens from 4 patients showing minimal and no specific findings in muscle biopsy were concurrently examined. We collected demographic data (gender, age of onset and age of diagnosis), gene mutated and specific mutations in order to correlate them with immunocytochemistry results.

Single immunocytochemistry. Five-micrometer serial sections from muscle specimens, stored in a freezer at $-80^{\circ}$, were incubated in acetone at $4^{\circ} \mathrm{C}$ for 10 minutes, washed in $0.05 \mathrm{~mol} / \mathrm{L}$ Tris-buffered saline $(\mathrm{pH}$ 7.5) for 15 minutes, and fixed for 30 minutes with a blocking reagent containing $2 \%$ bovine serum albumin and $5 \%$ normal goat serum or $5 \%$ horse serum. Sections were then incubated overnight at $4{ }^{\circ} \mathrm{C}$ with one of the following antibodies: goat polyclonal IgG against human MMP-9 (dilution 1:50; Santa Cruz, Santa Cruz, CA, USA) or rabbit polyclonal IgG against human MMP-2 (dilution 1:400; Chemicon, Temecula, CA, USA). These affinity-purified antibodies are well characterized and do not cross-react with each other. After being washed for 30 minutes in Tris-buffered saline, the sections were incubated with biotinylated goat anti-rabbit IgG for MMP-2 or biotinylated horse anti-goat IgG for MMP-9, followed by fluorescent isothiocyanate avidin D.

We analyzed qualitatively the MMP-2 and MMP-9 muscle immunoexpression from all patients, including controls by optic microscopy fluorescence in three different compartments - subsarcolemmal (SS), intracytoplasmic (IC), and perinuclear (PN) - classifying them as expressed (+) or no expressed (-). In order to correlate the histopathological and immunocytochemical findings, five-micrometer serial sections were also stained with hematoxilyn and eosin simultaneously.

\section{Statistical analysis}

To compare the immunostaining pattern and its correlation with demographic and genetic data, we performed the McNemar non-parametric test. For the tests, we used the SPSS 17.0 softwares for Windows. The significance level used for the tests was 5\% [11]. Qualitative variables were presented using absolute frequency and relative frequency. In order to compare MMP-2 and MPP-9 regulation among the genetic groups, the Chi-square test was used. The level of significance adopted in this analysis was $p<0.05$. The statistical program used was Stata version 11.0.

\section{Results}

Demographic features and molecular diagnosis from these patients are shown in Table 1. Among the patients, gender distribution was 11 (47.8\%) females and $12(52.2 \%)$ males. In our sample, 21 out 23 patients (91.3\%) were genetically identified $-D E S=9$ (39.1\%), ZASP=5 (21.7\%), FLNC=3 (13\%), MYOT=2 (8.7\%) and $C R Y A B=2(8.7 \%)$ - and in two we did not find a pathogenic variant (NMF) $(8.7 \%)$.

The age of diagnosis varied from 22 to 72 years (mean: 53.3 years; median: 56 years). The age of onset of symptoms ranged from 0 to 66 years (mean: 40.1 years; median: 41 years). The interval between the 
beginning of symptoms and the diagnosis varied from 0 to 34 years (mean: 13.3; median: 10).

The MMP-2 and MMP-9 muscle immunoexpression pattern distributions are presented in Table 1 and Graphic 1. It should be noted that both MMPs have a constitutive expression in endomysial blood vessels including control samples. There was no correlation between the immunoexpression pattern distribution and the duration of symptoms ( $p>0.05$ ). Most patients showed MMP-2 and MMP-9 expression $(+)$ in the IC compartment (95.7\% and $100 \%$, respectively). The control group showed no expressed (-) in all 4 samples. (Figure 1).

In terms of MMP-2 and MMP-9 expression, PN compartment showed a significant difference between MMP-2 expressed (82.6\%) and MMP-9 expressed (8.7\%) ( $p<0.001)$. In contrast, SS and IC compartments did not present statistical significance between MMP-2 and 9 expression (Graphic 1, Table 1) however, the protein aggregates showing expression (+) varied from one patient to another. In addition, the same protein aggregate was expressed by MMP-9 but not by MMP-2, and vice-versa in the same section (Figure $2 b)$. Also, the immunodistribution of MMPs were similar in most patients in relation to different genetic groups (FLNC, DES, MYOT, ZASP, CRYAB and NMF) ( $p>0.05)$.

\section{Discussion}

The pathogenesis and etiology of myofibrillar myopathy is not yet completed elucidated. Destruction of the myofibrillar network starting at the $z$-disk is supposed to be the first stage in the possible mechanism participating in the sarcomere's damage process.

Our study was a descriptive analysis about the behavior of MMP-2 and 9 in different compartments of the skeletal muscle fiber (Figure 1). Particularly, MMP-2 presented significant expression (+) in the PN compartment $(p<0.001)$ in relation to MMP-9. By contrast, MMP-9 expression $(+)$ was predominantly in the SS compartment demonstrating a possible influence of this gelatinase in ECM. In relation to IC compartment, our results were intriguing: both MMPs were expressed (+) in different regions of sarcoplasm. The same protein aggregate was expressed (+) by MMP-9 but not by MMP-2, and vice-versa in the same section (Figure $2 b$ ).

MMP-9 is normally expressed during early embryonic developmental while in normal adult tissues, it is not present suggesting that MMP-9 are increased upon tissue injury in order to repair damage, release growth factors and modulation of ECM. By contrast, the continuous expression of MMP-9 will contribute to inflammation and tissue destruction in different diseases [5]. Choi and Dalakas demonstrated that upregulation of MMP-2 and 9 in the muscle membrane of patients with s-IBM and PM may cause an active role in disarrangement of the integrity of the muscle fibers by the autoinvasive cytotoxic $T$ cells [7]. In addition, increased proinflammatory cytokines in mdx mice, appear to be some of the potential stimuli for up-regulation in MMP-9 levels in skeletal and cardiac muscle in muscular dystrophy [12].

In relation to MMP-2, it is constitutively expressed by many cell types including skeletal muscle and has a minimal regulation at the transcriptional level therefore, the regulation process takes place at the post- 
According to previous reports, MMP-2 is demonstrated in several subcellular compartments, including the thin and thick myofilaments, close to the $\mathrm{Z}$ disks of the cardiac sarcomere, the cytoskeleton, nuclei, mitochondria, and caveolae observed in cardiac sarcomere after injury induced by ischemia $[4,13]$.

In view of the aforementioned aspects of MMPs there would be another component of cell signaling that could be part of the protein aggregate deposit and MMPs expressed would be just an epiphenomenon? Or why not, those MMPS expressed (PN, IC and SS) could not be protection factor to muscle fiber?

We believe that both MMPs may participate actively in the pathological cascade leading to muscle membrane injury. MMP-9 comes from extracellular space into intracellular space and MMP-2 comes from intracellular space into extracellular space (Figures 1, 2a). Several intracellular processes are related to MMP-2 as cleaving specific proteins inside of myocytes thereafter reducing contractile function [4].

The mutations of known genes responsible for MFM will lead to an absence or dysfunction of coded proteins, causing mechanical instability of the sarcomere and, consequently, injury to the subcellular fiber compartments (ECM, sarcolemma, sarcoplasm, nucleus, and endoplasmic reticulum). The mechanical stress due to this instability would lead to MMP-2 upregulation with consequent destruction of the sarcomere, causing accumulation of proteins in the sarcoplasm (aggregates).

\section{Conclusion}

This study was the first one to analyze the role of MMPs in the pathogenesis of MFM. Our results showed that MMP-2 and 9 are expressed in different compartments of skeletal muscle while in control muscles they were not demonstrated. Therefore, they could participate in the pathomechanism of MFM, causing damage of sarcomere and deposition of protein aggregates.

More studies are necessary to clarify this new concept. Our study provides only preliminary qualitatively data about the role of MMPs in order to better understand the pathogenesis of this set of disorders.

\section{Abbreviations}

1. DES: desmin

2. DMD: Duchene muscular dystrophy

3. ECM: extracellular matrix

4. FLNC: filamin C

5. IC: intracytoplasmic

6. MMP: matrix metalloproteinase

7. MFM: myofibrillar myopathy

8. MYOT: myotilin 
9. NMF: no mutation found

10. PM: polymyositis

11. PN: perinuclear

12. sIBM: sporadic inclusion body myositis

13. SS: subsarcolemmal

14. Zasp (LDB3): LIM DOMAIN-BINDING 3

\section{Declarations}

\section{Ethics approval and consent to participate}

Written informed consent for DNA and RNA analysis was obtained from all the recruited patients when primary diagnostic procedures were performed, with explicit consent for future use for research purposes, according to the Declaration of Helsinki and the study was approved by our local Ethics Committee."

\section{Consent for publication}

Not applicable.

\section{Availability of data and material}

The datasets used and / or analyzed during the current study will be available from Professor Alves de Siqueira Carvalho on a reasonable request

\section{Competing interests}

None of the authors has any conflict of interest to disclose

\section{Funding}

Not applicable

\section{Acknowledgements}

Not applicable

\section{Authors' contributions}

All authors have contributed to the work, agree with the presented findings. The article has not been published before nor is being considered for publication in another journal. AASC and EL carried out the initial study, applied interventions, wrote the manuscript and coordinated the study. VGS, RC and APS participated in the study design and performed the statistical analyzes. All authors read and approved the final manuscript. 


\section{References}

1. Wang W, Schulze CJ, Suarez-Pinzon WL, Dyck JR, SawickiG, Schulz R. Intracellular action of matrix metalloproteinase-2 accounts for acute myocardial ischemia and reperfusion injury. Circulation. 2002;106: 1543-1549.

2. Sung MM, Schulz CG, Wang W, Sawicki G, Bautista-Lopez NL, Schulz R. Matrix metalloproteinase-2 degrades the cytoskeletal protein alpha-actinin in peroxynitrite mediated myocardial injury. J Mol Cell Cardiol. 2007; 43:429-36

3. DeCoux A, Lindsey ML, Villarreal F, Garcia RA, Schulz R. Myocardial matrix metalloproteinase-2: inside out and upside down. J Mol Cell Cardiol. 2014; 77:64-72

4. Schulz R. Intracellular Target of Matrix Metalloproteinase-2 in Cardiac Disease: Rationale and Therapeutic Approaches. Annu Rev Pharmacol Toxicol. 2007;47: 211-42

5. Ogura Y, Tajrishi MM, Sato S, Hindi SM, Kumar A. Therapeutic potential of matrix metalloproteinase in Duchenne muscular dystrophy. Front Cell Dev Biol. 2014; 2: 1-11.

6. Fukushima K, Nakamura A, Ueda H, Yuasa K, Yoshida K, Takeda S et al. Activation and localization of matrix metalloproteinase-2 and -9 in the skeletal muscle of the muscular dystrophy dog (CXMDJ). BMC Musculoskelet Disord. 2007 Jun 28; 8:54.

7. Choi YC, Dalakas MC. Expression of matrix metalloproteinases in the muscle of patients with inflammatory myopathies. Neurology 2000 Jan 11; 54(1): 65-71

8. Schoser BGH, Blottner D, Stuerenburg HJ. Matrix metalloproteinases in inflammatory myopathies: enhanced immunoreactivity near atrophic myofibers. Acta Neurol Scand 2002: 105: 309-13

9. Béhin A, Salort-Campana E, Wahbi K, Richard P, Carlier RY, Carlier P et al. Myofibrillar myopathies: State of the art, present and future challenges. Rev Neurol (Paris). 2015 Oct; 171(10): 715-29

10. Ferrer I; Olivé M. Molecular pathology of myofibrillar myopathies. Expert Rev Mol Med. 2008; 10 Sep 3;10: e25. doi: 10.1017/S1462399408000793.

11. Rosner B. Fundamentals of Biostatistics. Boston. PWS Publishers. Second edition. 584pp.

12. Acharyya S, Villalta SA, Bakkar N, Bupha-Intr T, Janssen PM, Carathers $M$ et al. Interplay of IKK/NFkappaB signaling in macrophages and myofibers promotes muscle degeneration in Duchenne muscular dystrophy. J. Clin. Invest.2007; 117, 889-901

13. Carlson BM, Faulkner JA. The regeneration of skeletal muscle fibers following injury: a review. Med Sci Sports Exerc. 1983; 15(3): 187-98.

\section{Table}

Due to technical limitations Table 1 is available as a download in the Supplementary Files.

\section{Supplementary File Legend}

Graphic 1: Comparative between different compartments of metalloproteinases 2 and 9. 


\section{Figures}
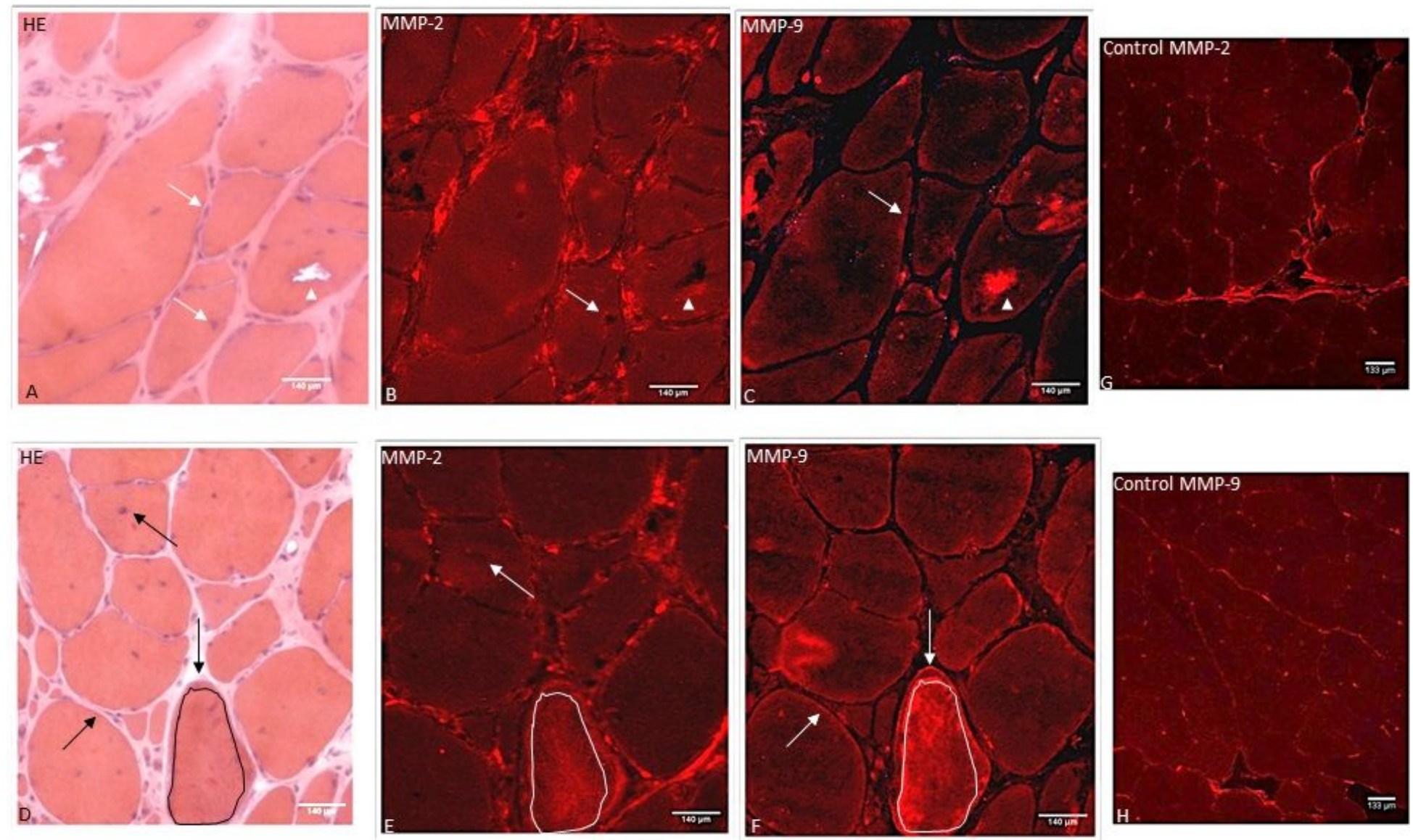

\section{Figure 1}

Immunolocalization of MMP-2 and MMP-9 Legend: A,B,C: patient 9, MYOT mutation: HE stain; MMP-2 expressed perinuclear (PN) (arrow) and intracytoplasmic (IC) (arrow head); MMP-9 expressed subsarcolemmal (SS) (arrow) and IC (arrow head); D,E,F: patient 14: DES mutation: HE stain;; MMP-2 expressed IC (circle) and PN (arrow); MMP-9 expressed SS (arrow) and IC (circle). G, H: normal control 
Figure 2. Schematic view of the sarcomere structure and role of MMP-2 and MMP-9.

2a.

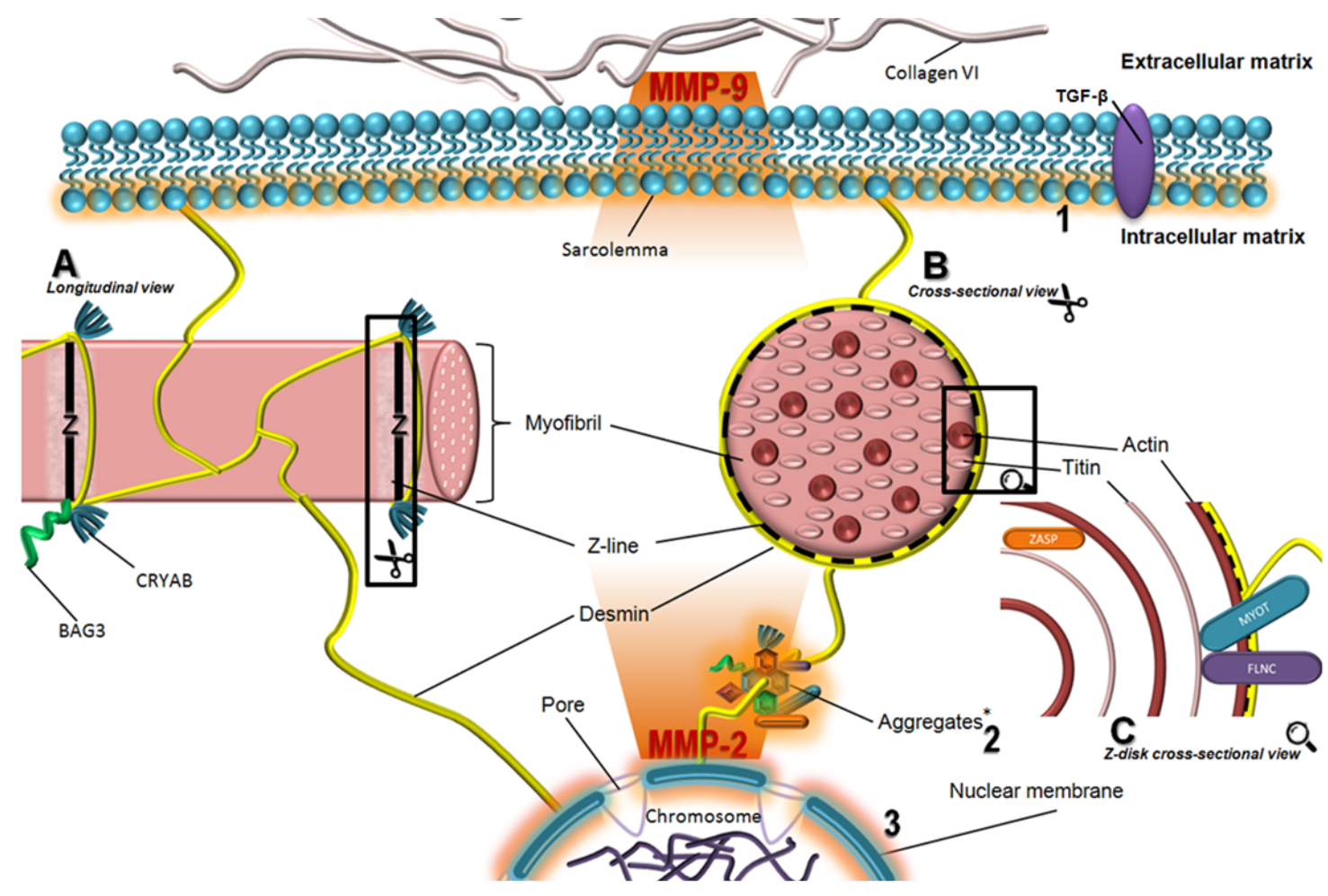

$2 b$.
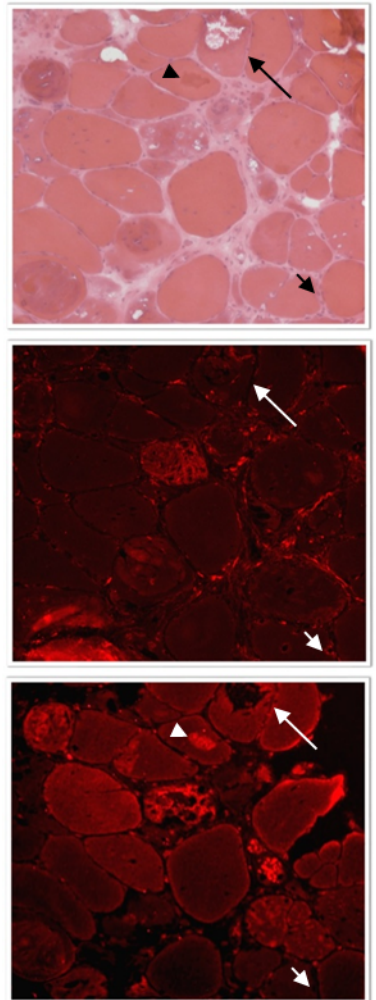

Figure 2

Schematic view of the sarcomere structure and role of MMP-2 and MMP-9. Legend: 2a. Schematic view of the sarcomere structure and its relationship with proteins of Z-line and presence of aggregates due a mutation (DES, ZASP, MYOT, BAG3, FLNC); 2b. MMP2- and MMP9 immunodistribution and its correlation with pathological findings in H\&E stain (2b-1). MMP-2 upregulation PN (thick arrow) and IC (arrow); MMP9 upregulation IC (including aggregates) (arrow head) and SS (thick arrow).

\section{Supplementary Files}

This is a list of supplementary files associated with this preprint. Click to download.

- Table1.pdf

- AlziraCarvalhoGraphic01MMP.tif 